\title{
Fratture di polso: cenni storici e introduzione
}

$\mathrm{N}$

ella totalità delle fratture, quelle dell'estremità distale del radio sono il 20 per

cento e, di queste, il 50 per cento interessa la radio-carpica e la radio-ulnare.

Da Ippocrate (469-356 a.C.) fino alla metà del Settecento sono state scambiate, nella maggioranza dei casi, per lussazioni e, con una certa frequenza, il trattamento consisteva nell'amputazione della mano: o per sopraggiunta suppurazione o per gangrena, esito di bendature troppo serrate, o per esiti algodistrofici che rendevano la mano inservibile e dolorosa. Sul moncone, talora, era applicata una rudimentale protesi.

Come si legge negli antichi trattati, più che dell'anatomia patologica gli Autori si preoccupavano di descrivere la tecnica dell'amputazione (vedasi, per esempio, Armamentarium chirurgicum bipartum di G. Sultato, 1666). Prima della descrizione di Colles (1814), era stato Pouteau (1783) a riferire di un caso di frattura di polso, ma si deve sopratutto al barone Dupuytren (1777-1835) il merito di aver dato una svolta decisiva all'interpretazione del quadro anatomo-patologico delle fratture di polso. Con Dupuytren iniziava una nuova era nel trattamento di queste fratture, finalmente definite: cercando il riallineamento dei monconi con la trazione, contrastando la tumefazione con l'applicazione di sanguisughe, con cataplasmi e/o con acqua vegeto-minerale e applicando apparecchi di contenzione appositi (apparecchio cubitale dell'HôtelDieu, ove Dupuytren aveva ricoperto il ruolo di capo di Anatomia prima di arrivare alla direzione della Chirurgia).

In seguito, nel 1846, Vanzetti introdusse l'uso delle bende di colla, sostituite da quelle gessate nel 1878 per merito di Mathiesen. Agli inizi del Novecento, Cotton-Loder codificava la posizione della riduzione con apparecchio gessato, che sopravisse fin verso l'inizio della seconda metà del secolo. Negli ultimi 30 anni si sono sviluppati materiali e tecniche più sofisticati:

- il fissatore esterno, che permette la distrazione e la traslazione palmare;

- impianti e placche a basso profilo per la fissazione interna;

- l'ausilio dell'artroscopia per la riduzione;

- le tecniche di innesto osseo e relativi sostituti.

Il tutto ha contribuito a migliorare la stabilità delle fratture e i risultati. Attualmente qual è il razionale del trattamento delle fratture di polso? 
Negli articoli che seguiranno vengono descritte le indicazioni e le metodiche condivise o condivisibili secondo la tipologia delle fratture. Ma, per una visione d'insieme riassuntiva, concludo queste note con uno schema di algoritmo utile per orientare verso il tipo di trattamento delle fratture di polso.

\section{Cugola}

Ospedale di Peschiera del Garda (VR)

\section{Frattura}

\section{Trattamento}

I. Extra-articolare Apparecchio gessato composta

II. Extra-articolare scomposta

a) Riduzione chiusa $>$ stabile - Apparecchio gessato + (fili di Kirschner)

b) Riduzione chiusa > instabile - FE + (fili di Kirschner - opzione placca)

III. Intra-articolare Apparecchio gessato \pm fili di Kirschner (opzione placca) composta

IV. Intra-articolare scomposta

a) Riducibile-stabile $>$ apparecchio gessato \pm fili di Kirschner

b) Riducibile-instabile $>$ riduzione aperta + placca (eventualmente fili di Kirschner)

c) Irriducibile-instabile $>$ riduzione aperta + placca-fili-FE

d) complessa-irriducibile-instabile $>\mathrm{FE}$

$>$ Riduzione aperta + fili di Kirschner/innesto/placca

$>$ Riparazione delle lesioni associate 